\title{
The Impact of Mobile Access to the Internet on Information Search Completion Time and Customer Conversion
}

\author{
Ushio Sumita, Jinshui Zuo \\ Graduate School of Systems and Information Engineering, University of Tsukuba, \\ 1-1-1,Tenoudai, Tsukuba, Ibaraki, 305-8573, Japan \\ (E-mail address: sumita@sk.tsukuba.ac.jp;ksa@sk.tsukuba.ac.jp)
}

\begin{abstract}
One of the advantages of mobile access to the Internet can be found in that it allows one to conduct information search in a ubiquitous manner. Accordingly, the time for net surfing required to gain necessary information through the Internet is reduced when mobile access is combined with ordinary PC access. As mobile devices continue to advance rapidly, it becomes quite important to find a way to assess the impact of mobile access to the Internet on the performance of e-commerce. Among such performance indicators, of particular interest is the conversion rate, where a customer takes some desirable action at the website, for example, by becoming a member, placing an order and so on. The purpose of this paper is to develop and analyze a mathematical model for describing the information search process through the Internet with or without the mobile access. The analytical framework that we propose in this article enables one to assess the impact of mobile access on information search completion time and the conversion rate. We provide numerical examples to demonstrate the effectiveness of the computational procedures that we developed in this research.
\end{abstract}

Key words: conversion rate, information search completion time, mobile access, PC access

\section{Introduction}

Independent studies have shown that mobile access to the Internet has been evolving quite rapidly. According to Juniper Research (2008), the num- 
ber of mobile Internet users will grow from 577 million in 2008 to 1,700 million in 2013 in the world. iSuppli (2006) reports that the number of mobile phone users would reach 4 billion in the world by 2010. Mobile phone access to the Internet is widespread in Japan and Korea, while PDAs are mostly used in the United States for the mobile access to the Internet (Roto 2005). Khan et al. (2008) point out that, although penetration in the United States is high with $84 \%$ of Americans using mobile phones, the mobile search market is in the early adoption stage. However, according to Neilsen (2008) and Osterman (2007), this industry is expected to grow drastically and will become important in marketing and lead to the enhancement of workforce productivity.

Table 1 compares the features of mobile and PC-based access to the Internet. It shows that mobile access to the Internet is still rather limited. However, the advantage of mobile access over PC-based access is that it support ubiquitous information search. As a result, the search time for information is likely to be reduced when mobile and PC-based accesses to the Internet are combined.

Table 1. Characteristics of Mobile and PC-based Access

\begin{tabular}{|c|c|c|}
\hline & Mobile Access & PC-based Access \\
\hline Screen Size & Small & Large \\
\hline Nonumber of Windows & Single & Multiple \\
\hline Navigation & Slow & Fast \\
\hline Speed & Low & High \\
\hline Size of Message & Small & Big \\
\hline Mobility & Yes & No \\
\hline
\end{tabular}

The impact of mobile access to the Internet on e-businesses has been addressed by many researchers including Roto (2005), Kim (2006) and Chae and Kim (2003) to name only a few. In addition, Barwise (2001) and Hammond (2001) discuss the future trends in m-commerce. Wu and Hisa (2004) propose a hypercube model for analyzing e-commerce innovation and impact including m-commerce. Siau et al. (2004), and Park and Fader (2004) analyze m-commerce from the perspective of consumers, while Thompson and Garbacz (2007) discuss the effects of e-commerce and m-commerce on global productive efficiency. Roto (2006) discusses how information search on a mobile phone differs from information search on a PC with Internet access. Okazaki and Hirose (2009) examine media displacement that have occurred due to changes in traditional media, and mobile and PC-based access to 
the Internet. These works involve mostly empirical and qualitative analysis. No mathematical models appear to exist in the literature for analyzing how mobile access facilitates information search on the Internet.

In e-commerce, a key performance indicator is the conversion rate, signifying when a customer takes a desirable e-commerce-related action at a website, for example, by becoming a member, placing an order and so on. Before deciding whether to do these things, individual customers typically gather information through the Internet. To analyze conversion rates over time, it is appropriate to measure how long it takes for customers to complete their search for information. The purpose of this paper is to develop and analyze a mathematical model for understanding the impact of mobile access to the Internet on information search completion time. This, in turn, will enable the assessment of how mobile access to the Internet enhances the conversion rate.

We will consider an e-commerce website in which individual customers search for information on the website and on the Internet at large, before deciding whether to take an action. This information search will be completed when the cumulative amount of information gathered through net surfing exceeds some pre-specified level $z$. When the search is complete, each customer will take action with probability $p$. If no action is taken, the corresponding probability is $1-p$. With $N$ customers at time 0 , an issue of interest is how many of them will finish searching for information and take an action by time $t$.

The structure of the paper is as follows. In Section 2, we present a mathematical model to describe the information search process on the Internet with and without the mobile access. Section 3 analyzes information search completion time in terms of PC-based Internet access only $\left(T_{P C: z}\right)$, mobile access only $\left(T_{M: z}\right)$, and with both mobile and PC-based access $\left(T_{B: z}\right)$. We derive the double Laplace transforms of their probability density functions with respect to the threshold level and information search completion time. We also derive their means and variances. In Section 4, we analyze the double transforms under the assumption of exponential information gathering and deterministic search time. In Section 5, we present numerical examples to demonstrate the effectiveness of our computational procedures for assessing the impact of mobile access on information search completion time and the customer conversion rate. Section 6 concludes. 


\section{Model Description}

In this section, we develop a mathematical model for describing a customer's search process with and without mobile access to the Internet. Let $Y_{j}$ be the random duration available for a customer's search on the $j$-th day, $j=1,2, \cdots$, where $Y_{j}$ are assumed to be independently and identically distributed (i.i.d.). We call $Y_{j}$ the $j$-th search period. In what follows, we suppress non-search time along the time axis. So "at time $t$ " means "at the point in time" at which the cumulative search time has reached $t$.

A customer is of Type PC if the customer only has PC-based access to the Internet. Let $X_{1}(j)$ be the amount of information gained by the customer through PC-based access during the $j$-th search period. Naturally, the amount of information that can be gathered will depend on the amount of time available for search. However, search in one period will be independent of search in another period. Accordingly, throughout the paper, we assume that $X_{1}(j)$ and $Y_{j}$ are correlated for each $j$, but the vectors $\left[X_{1}(j), Y_{j}\right]$ are i.i.d. with respect to $j$. Information search is completed whenever the cumulative amount of information gained exceeds a pre-specified level $z$. More specifically, let $N(t)$ be the renewal process associated with $\left(Y_{j}\right)_{j=1}^{\infty}$, and define

$$
S_{P C}(t) \stackrel{\text { def }}{=} \sum_{j=1}^{N(t)} X_{1}(j)
$$

The information search completion time $T_{P C: z}$ for a Type PC customer given the threshold level $z$ is then obtained as

$$
T_{P C: z} \stackrel{\text { def }}{=} \inf \left\{t: S_{P C}(t)>z\right\}
$$

A customer is of Type $\mathrm{M}$ if the customer only has mobile access to the Internet. Let $X_{2}(j)$ be the amount of information gained by a Type M customer with mobile access during the $j$-th search period. The cumulative amount of information gained by a Type M customer through search by time $t$ is then given by

$$
S_{M}(t) \stackrel{\text { def }}{=} \sum_{j=1}^{N(t)} X_{2}(j)
$$


The information search completion time $T_{M: z}$ for a Type $\mathrm{M}$ customer given the threshold level $z$ is obtained as

$$
T_{M: z} \stackrel{\text { def }}{=} \inf \left\{t: S_{M}(t)>z\right\} .
$$

Type B customers are those who utilize the Internet via PCs or mobile devices. Let $X_{1}(j)$ and $X_{2}(j)$ be the amount of information gathered by a Type B customer with PC-based or mobile access during the $j$-th search period $Y_{j}$. As before, it is assumed that $\underline{X}(j)=\left[X_{1}(j), X_{2}(j)\right]$ and $Y_{j}$ are correlated for each $j$, but the vectors $\left[\underline{X}(j), Y_{j}\right]$ are i.i.d. with respect to $j$. Let $S_{B}(t)$ be the cumulative amount of information gathered through Internet search by a Type B customer by time $t$ :

$$
S_{B}(t) \stackrel{\text { def }}{=} \sum_{j=1}^{N(t)} Z_{B}(j) \quad ; \quad Z_{B}(j)=X_{1}(j)+X_{2}(j)
$$

The search completion time $T_{B: z}$ for a type $\mathrm{B}$ customer given a threshold level $z$ is then given by

$$
T_{B: z} \stackrel{\text { def }}{=} \inf \left\{t: S_{B}(t)>z\right\} .
$$

Of interest are the distributions of $T_{P C: z}, T_{M: z}$ and $T_{B: z}$ enable one to assess the impact of mobile access to the Internet on information search completion time. This will allow us to evaluate how the conversion rate improves in the presence of mobile access.

\section{Analysis of Information Search Completion Time}

The information search completion times $T_{P C: z}$ and $T_{M: z}$ for Type PC and Type M customers have a structure of the cumulative shock model associated with a renewal sequence discussed by Sumita and Shanthikumar (1985). For Type B customers, however, the model in Sumita and Shanthikumar (1985) should be modified so as to accommodate a sequence of bivariate shocks $\underline{X}(j)$ associated with a renewal sequence $Y_{j}$. The purpose of this section is to derive the Laplace transform of the probability density function (p.d.f) of $T_{B: z}$ along with its mean and variance. We confirm that the counterparts for $T_{P C: z}$ and $T_{M: z}$ originally given in Sumita and Shanthikumar (1985) will be obtained as a special case. 
Let $F_{\underline{X}, Y}\left(x_{1}, x_{2}, y\right)$ be the joint distribution function of the vector process $\underline{X}(j)=\left[\bar{X}_{1}(j), X_{2}(j)\right]$ and $Y_{j}$ defined by

$$
F_{\underline{X}, Y}\left(x_{1}, x_{2}, y\right)=P\left[X_{1}(j) \leq x_{1}, X_{2}(j) \leq x_{2}, Y_{j} \leq y\right] \quad .
$$

It is assumed that $F_{\underline{X}, Y}\left(x_{1}, x_{2}, y\right)$ is absolutely continuous with joint p.d.f $f_{\underline{X}, Y}\left(x_{1}, x_{2}, y\right)$ satisfying

$$
F_{\underline{X}, Y}\left(x_{1}, x_{2}, y\right)=\int_{0}^{x_{2}} \int_{0}^{x_{1}} \int_{0}^{y} f_{\underline{X}, Y}\left(x_{1}^{\prime}, x_{2}^{\prime}, y^{\prime}\right) d y^{\prime} d x_{1}^{\prime} d x_{2}^{\prime} .
$$

From Equation (5), the joint distribution function of $Z_{B}(j)$ and $Y_{j}$ denoted by $G_{Z_{B}, Y}(z, y)$ can be obtained as

$$
\begin{aligned}
G_{Z_{B}, Y}(z, y) & \stackrel{\text { def }}{=} P\left[Z_{B}(j) \leq z, Y_{j} \leq y\right]=P\left[X_{1}(j)+X_{2}(j) \leq z, Y_{j} \leq y\right] \\
& =\int_{0}^{y}\left\{\int_{0}^{z}\left\{\int_{0}^{z^{\prime}} f_{\underline{X}, Y}\left(z^{\prime}-x, x, y^{\prime}\right) d x\right\} d z^{\prime}\right\} d y^{\prime}
\end{aligned}
$$

Let $g_{Z_{B}, Y}(z, y)$ be the joint p.d.f of $Z_{B}(j)$ and $Y_{j}$. One then has

$$
g_{Z_{B}, Y}(z, y) \stackrel{\text { def }}{=} \frac{\partial^{2}}{\partial z \partial y} G_{Z_{B}, Y}(z, y)=\int_{0}^{z} f_{\underline{X}, Y}(z-x, x, y) d x .
$$

Throughout the paper, Laplace transforms with respect to $t, z$ or both are denoted by a circumflex or a double circumflex. The Laplace transform of $g_{Z_{B}, Y}(z, y)$ is denoted accordingly by

$$
\hat{\hat{g}}_{Z_{B}, Y}(w, s) \stackrel{\text { def }}{=} \int_{0}^{\infty} \int_{0}^{\infty} e^{-s y-w z} g_{Z_{B}, Y}(z, y) d z d y \quad .
$$

For notational convenience, the following functions are also introduced.

$$
\begin{aligned}
f_{X_{1}}\left(x_{1}\right) & =\int_{0}^{\infty} \int_{0}^{\infty} f_{\underline{X}, Y}\left(x_{1}, x_{2}, y\right) d x_{2} d y \\
f_{X_{2}}\left(x_{2}\right) & =\int_{0}^{\infty} \int_{0}^{\infty} f_{\underline{X}, Y}\left(x_{1}, x_{2}, y\right) d x_{1} d y \\
f_{Y}(y) & =\int_{0}^{\infty} \int_{0}^{\infty} f_{\underline{X}, Y}\left(x_{1}, x_{2}, y\right) d x_{1} d x_{2} \\
f_{X_{1}, Y}\left(x_{1}, y\right) & =\int_{0}^{\infty} f_{\underline{X}, Y}\left(x_{1}, x_{2}, y\right) d x_{2} \\
f_{X_{2}, Y}\left(x_{2}, y\right) & =\int_{0}^{\infty} f_{\underline{X}, Y}\left(x_{1}, x_{2}, y\right) d x_{1} .
\end{aligned}
$$




$$
\begin{aligned}
\hat{\varphi}_{X_{1}}(w) & \stackrel{\text { def }}{=} \int_{0}^{\infty} e^{-w x_{1}} f_{X_{1}}\left(x_{1}\right) d x_{1} \\
\hat{\varphi}_{X_{2}}(w) & \stackrel{\text { def }}{=} \int_{0}^{\infty} e^{-w x_{2}} f_{X_{2}}\left(x_{2}\right) d x_{2} \\
\hat{\varphi}_{Y}(s) & \stackrel{\text { def }}{=} \int_{0}^{\infty} e^{-s y} f_{Y}(y) d y \\
\hat{\hat{\varphi}}_{X_{1}, Y}(w, s) & \stackrel{\text { def }}{=} \int_{0}^{\infty} \int_{0}^{\infty} e^{-w x_{1}-s y} f_{X_{1}, Y}\left(x_{1}, y\right) d x_{1} d y \\
\hat{\hat{\varphi}}_{X_{2}, Y}(w, s) & \stackrel{\text { def }}{=} \int_{0}^{\infty} \int_{0}^{\infty} e^{-w x_{2}-s y} f_{X_{2}, Y}\left(x_{2}, y\right) d x_{2} d y
\end{aligned}
$$

Let the distribution functions of $S_{B}(t)$ and $T_{B: z}$ be defined by

$$
V_{B}(z, t)=P\left[S_{B}(t)<z\right] \quad ; \quad W_{B}(z, t)=P\left[T_{B: z} \leq t\right]
$$

respectively. Absolute continuity of $F_{\underline{X}, Y}\left(x_{1}, x_{2}, y\right)$ assures that $T_{B: z}$ has the probability density function given by

$$
w_{B}(z, t)=\frac{\partial}{\partial t} W_{B}(z, t)
$$

We define

$$
\begin{gathered}
\hat{\hat{V}}_{B}(w, s)=\int_{0}^{\infty} \int_{0}^{\infty} e^{-s t-w z} V_{B}(t, z) d z d t \\
\hat{\hat{w}}_{B}(w, s)=\int_{0}^{\infty} \int_{0}^{\infty} e^{-s t-w z} w_{B}\left(z, t^{\prime}\right) d z d t^{\prime}
\end{gathered}
$$

There is a dual relationship between $S_{B}(t)$ and $T_{B: z}$ specified by

$$
V_{B}(z, t)=P\left[S_{B}(t)<z\right]=P\left[T_{B: z}>t\right]=\bar{W}_{B}(z, t),
$$

where $\bar{W}_{B}(z, t)=1-W_{B}(z, t)$ is the survival function of $T_{B: z}$. The following theorem then holds.

Theorem 1. (Double Laplace Transform of Distribution of Cumulative Gathered Information through both PC Access and Mobile Access)

$$
\hat{\hat{V}}_{B}(w, s)=\frac{1-\hat{\varphi}_{Y}(s)}{w s\left\{1-\hat{\hat{g}}_{Z_{B}, Y}(w, s)\right\}} \quad, \quad \operatorname{Re}(s) \geq 0 .
$$


Proof. Since $V_{B}(z, t)$ is the probability that the cumulative amount of $Z_{B}(j)$ has not exceeded the level $z$ for $0 \leq j \leq N(t)$, by conditioning on the first renewal time $Y_{1}$ and using the regenerative property of the paired process $\left[\underline{X}(j), Y_{j}\right]$ at $Y_{1}$, one sees that

$$
V_{B}(z, t)=\bar{F}_{Y}(t)+\int_{0}^{t} \int_{0}^{z} g_{Z_{B}, Y}(x, y) V_{B}(z-x, t-y) d x d y .
$$

By taking the Laplace transform of both sides of Equation (27) with respect to $z$ and $t$, it can be seen that

$$
\hat{\hat{V}}_{B}(w, s)=\frac{1-\hat{\varphi}_{Y}(s)}{w s}+\hat{\hat{g}}_{Z_{B}, Y}(w, s) \hat{\hat{V}}_{B}(w, s)
$$

This equation can be solved for $\hat{\hat{V}}_{B}(z, s)$, completing the proof.

Information search completion time $T_{B: z}$ has a dual relationship with $S_{B}(t)$ given in Equation (26). The Laplace transform $\hat{\hat{w}}_{B}(w, s)=E\left[e^{-s T_{B: z}}\right]$ is then easily found from Theorem 1.

Theorem 2. (Double Laplace Transform of Distribution of Search Completion Time through both PC Access and Mobile Access)

$$
\hat{\hat{w}}_{B}(w, s)=\frac{\hat{\varphi}_{Y}(s)-\hat{\hat{g}}_{Z_{B}, Y}(w, s)}{w\left\{1-\hat{\hat{g}}_{Z_{B}, Y}(w, s)\right\}} \quad, \quad \operatorname{Re}(s) \geq 0 .
$$

Proof. From (26), one finds that $\hat{\hat{V}}_{B}(w, s)=\frac{\frac{1}{w}-\hat{\hat{w}}_{B}(w, s)}{s}$, so that $\hat{\hat{w}}_{B}(w, s)=$ $\frac{1}{w}-s \hat{\hat{V}}_{B}(w, s)$. The theorem now follows.

By differentiating $\hat{\hat{w}}_{B}(w, s)$ with respect to $s$ once or twice at $s=0$ and taking the inverse with respect to $w$, the mean and the variance of $T_{B: z}$ can be obtained as given in the following corollary. The proof is omitted.

Corollary 3. (Mean and Variance of Search Completion Time through both PC Access and Mobile Access)

Let $\eta_{Y}$ and $\eta_{Y_{2}}$ denote the mean and the second moment of $Y_{j}$, and let $H_{B}(z)$ be the renewal function associated with $Z_{B}$, that is, $H_{B}(z) \stackrel{\text { def }}{=} \sum_{n=1}^{\infty} G_{Z_{B}}^{(n)}(z)$, 
where $G_{Z_{B}}^{(n)}(z)=\int_{0}^{z} G_{Z_{B}}^{(n-1)}(z) d G_{Z_{B}}(z)$ for $n \geq 2$ starting with $G_{Z_{B}}^{(1)}(z)=$ $G_{Z_{B}}(z)=G_{Z_{B}}(z, \infty)$. One then finds that

a) $E\left[T_{B: z}\right]=\eta_{Y}\left\{1+H_{B}(z)\right\}$

b) $\operatorname{Var}\left[T_{B: z}\right]=\eta_{Y_{2}}\left\{1+H_{B}(z)\right\}+2 \eta_{Y} \int_{0}^{z} G_{Z_{B}}^{(n)}(z-x) E[Y \mid Z=x] d G_{Z_{B}}(x)$ $-\eta_{Y}^{2}\left\{1+H_{B}(z)\right\}^{2}$.

The counterpart of Theorem 2 for the search completion time $T_{P C: z}$ for Type PC customers or $T_{M: z}$ for Type $\mathrm{M}$ customers can be obtained by setting $X_{2}(j)=0$ or $X_{1}(j)=0$ respectively. More specifically, let the distribution function and the probability density function of $T_{P C: z}$ and $T_{M: z}$ be defined by

$$
W_{P C}(z, t)=P\left[T_{P C: z} \leq t\right] \quad ; \quad W_{M}(z, t)=P\left[T_{M: z} \leq t\right]
$$

and

$$
w_{P C}(z, t)=\frac{\partial}{\partial t} W_{P C}(z, t) \quad ; \quad w_{M}(z, t)=\frac{\partial}{\partial t} W_{M}(z, t) \quad,
$$

with the correspoding Laplace transforms with respect to $t$ and $z$ given by

$$
\begin{aligned}
\hat{\hat{w}}_{P C}(w, s) & =\int_{0}^{\infty} \int_{0}^{\infty} e^{-s t-w z} w_{P C}\left(z, t^{\prime}\right) d z d t^{\prime} \\
\hat{\hat{w}}_{M}(w, s) & =\int_{0}^{\infty} \int_{0}^{\infty} e^{-s t-w z} w_{M}\left(z, t^{\prime}\right) d z d t^{\prime}
\end{aligned}
$$

From Equations (20) and (21), the following theorem holds by replacing $\hat{\hat{g}}_{Z_{B}, Y}(w, s)$ in Theorem 2 by $\hat{\hat{\varphi}}_{X_{1}, Y}(w, s)$ and $\hat{\hat{\varphi}}_{X_{2}, Y}(w, s)$, respectively.

Theorem 4. (Double Laplace Transform of Distribution of Search Completion Time through PC Access Only and That through Mobile Access Only)

$$
\begin{array}{ll}
\text { a) } \quad \hat{\hat{w}}_{P C}(w, s)=\frac{\hat{\varphi}_{Y}(s)-\hat{\hat{\varphi}}_{X_{1}, Y}(w, s)}{w\left\{1-\hat{\hat{\varphi}}_{X_{1}, Y}(w, s)\right\}} \quad, \quad \operatorname{Re}(s) \geq 0 \\
\text { b) } \quad \hat{\hat{w}}_{M}(w, s)=\frac{\hat{\varphi}_{Y}(s)-\hat{\hat{\varphi}}_{X_{2}, Y}(w, s)}{w\left\{1-\hat{\hat{\varphi}}_{X_{2}, Y}(w, s)\right\}} \quad, \quad \operatorname{Re}(s) \geq 0
\end{array}
$$

By differentiating $\hat{\hat{w}}_{P C}(w, s)$ and $\hat{\hat{w}}_{M}(w, s)$ with respect to $s$ once or twice at $s=0$ and then inverting the results with respect to $w$, the next corollary follows as before. 
Corollary 5. (Mean and Variance of Search Completion Time through PC Access Only and Those through Mobile Access Only)

Let $\eta_{Y}$ and $\eta_{Y_{2}}$ be as in Corollary 3, and let $H_{P C}(z)$ and $H_{M}(z)$ be the renewal functions associated with $X_{1}$ and $X_{2}$ respectively. One then finds that

a) $E\left[T_{P C: z}\right]=\eta_{Y}\left\{1+H_{P C}(z)\right\}$

b) $E\left[T_{M: z}\right]=\eta_{Y}\left\{1+H_{M}(z)\right\}$

c) $\operatorname{Var}\left[T_{P C: z}\right]=\eta_{Y_{2}}\left\{1+H_{P C}(z)\right\}+2 \eta_{Y} \int_{0}^{z} F_{X_{1}}^{(n)}(z-x) E[Y \mid Z=x] d F_{X_{1}}(x)$ $-\eta_{Y}^{2}\left\{1+H_{P C}(z)\right\}^{2}$

d) $\operatorname{Var}\left[T_{M: z}\right]=\eta_{Y_{2}}\left\{1+H_{M}(z)\right\}+2 \eta_{Y} \int_{0}^{z} F_{X_{2}}^{(n)}(z-x) E[Y \mid Z=x] d F_{X_{2}}(x)$ $-\eta_{Y}^{2}\left\{1+H_{M}(z)\right\}^{2}$

We note that Theorem 4 and Corollary 5 coincide with Theorem 1.A2 and Corollary 1.A3 of Sumita and Shanthikumar (1985).

In principle, $W_{B}(z, t), W_{P C}(z, t)$ and $W_{M}(z, t)$ can be evaluated by inverting $\hat{\hat{w}}_{B}(w, s) / s, \hat{\hat{w}}_{P C}(w, s) / s$ and $\hat{\hat{w}}_{M}(w, s) / s$ with respect to both $w$ and $s$ based on Theorems 2 and 4 . Given the numbers of customers in Type B, Type PC and Type M, denoted by $N_{B}, N_{P C}$ and $N_{M}$ respectively, it then becomes possible to find the distribution of the number of customers who have made a conversion by time $t$. More specifically, for $\operatorname{Var} \in\{B, P C, M\}$, let $K_{V a r}(t)$ be the number of Type Var customers who have made a conversion by time $t$ and define

$$
P_{V a r}(k, t) \stackrel{\text { def }}{=} P\left[K_{V a r}(t)=k\right] \quad, k=0,1, \cdots N_{V a r} .
$$

It can be seen that the following theorem holds. The proof is omitted.

Theorem 6. (Distribution of Number of Conversions by Time t) For $\operatorname{Var} \in\{B, P C, M\}$ and $k=0,1, \cdots N_{\text {Var }}$, one has

$$
P_{\text {Var }}(k, t)=\left(\begin{array}{c}
N_{V a r} \\
k
\end{array}\right)\left\{p \cdot W_{\text {Var }}(z, t)\right\}^{k} \cdot\left\{1-p \cdot W_{\text {Var }}(z, t)\right\}^{N_{B_{V a r}}-k} .
$$

Considering that a website manager may be interested in the time needed to achieve a desired level of conversion rate, we introduce the minimal time needed to have $K$ customers with conversion by time $t$. Formally, we define

$$
\tau_{V a r}(K) \stackrel{\text { def }}{=} \inf \left\{t: K_{V a r}(t) \geq K\right\}
$$


and the associated survival function is denoted by

$$
\bar{D}_{V a r}(K, t)=P\left[\tau_{V a r}(K)>t\right] .
$$

The following theorem is then immediate from Theorem 6 .

Theorem 7. (Distribution of Time Required to Achieve $K$ Conversions) For Var $\in\{B, P C, M\}$ and $0<K \leq N_{\text {Var }}$, one has

$$
\bar{D}_{V a r}(K, t)=\sum_{k=0}^{K-1} P_{V a r}(k, t)
$$

In general, the double inversion of $\hat{\hat{w}}_{\text {Var }}(w, s)$ is laborious and cumbersome. In the next section, we overcome this difficulty by assuming that the time period available for Internet search in a day is deterministic, that is, $Y_{j}=T$ for all $j$, and the amount of information gathered through Internet search consists of two parts: a part which is proportional to $T$ and another part which is exponentially distributed and independent of $T$.

\section{Inversion of Double Laplace Transforms for Exponential Infor- mation Gathering and Deterministic Search Time}

In this section, we evaluate the distribution function of the information search completion time for Type B, Type PC and Type M customers explicitly based on the results of Section 3. This provides a computational vehicle for assessing the impact of mobile access to the Internet on the conversion rate. We will address this issue in Section 5. For this purpose, we assume that both $X_{1}(j)$ and $X_{2}(j)$ consist of two parts: a part independent of $Y_{j}$ and another part proportional to $Y_{j}$. The former parts for $X_{1}(j)$ and $X_{2}(j)$ are denoted by $\hat{X}_{1}(j)$ and $\hat{X}_{2}(j)$ respectively. More formally, we define

$$
X_{1}(j)=\hat{X}_{1}(j)+\alpha_{P C} Y_{j} \quad ; \quad X_{2}(j)=\hat{X}_{2}(j)+\alpha_{M} Y_{j},
$$

where $\hat{X}_{1}(j), \hat{X}_{2}(j)$ and $Y_{j}$ are independent of each other, but $X_{1}(j)$ and $X_{2}(j)$ are not independent because of sharing $Y_{j}$.

It is natural to assume that the amount of information gathered with PC-based Internet search will be stochastically larger than that gathered via Internet search on a mobile device. This is because mobile access is often limited in time compared with PC-based access. Furthermore, the screen size 
and the memory capacity of mobile devices is also more limited than those of PCs. Accordingly, we assume that

$$
\hat{X}_{1}(j) \succ_{s t .} \hat{X}_{2}(j) \text { and } \alpha_{P C} \geq \alpha_{M}
$$

where, for nonnegative random variables $V$ and $W, V \succ_{\text {st. }} W$ means that $P[V>x] \geq P[W>x]$ for all $x \geq 0$. Although this assumption is not essential, we nevertheless adopt it.

Let the distribution functions of $\hat{X}_{1}$ and $\hat{X}_{2}$ be denoted by $F_{\hat{X}_{1}}(x)$ and $F_{\hat{X}_{2}}(x)$ respectively, and let $F_{\underline{X}, Y}\left(x_{1}, x_{2}, y\right)$ be the joint distribution function of $\underline{X}$ and $Y$. From Equation (35), by conditioning on $Y$, one finds that

$$
\begin{array}{r}
F_{\underline{X}, Y}\left(x_{1}, x_{2}, y\right)=P\left[X_{1} \leq x_{1}, X_{2} \leq x_{2}, Y \leq y\right] \\
=\int_{0}^{m i n\left\{y, \frac{x_{1}}{\alpha_{P C}}, \frac{x_{2}}{\alpha_{M}}\right\}} F_{\hat{X}_{1}}\left(x_{1}-\alpha_{P C} \tau\right) F_{\hat{X}_{2}}\left(x_{2}-\alpha_{M} \tau\right) f_{Y}(\tau) d \tau .
\end{array}
$$

From (8), it then follows that

$$
\begin{aligned}
f_{\underline{X}, Y}\left(x_{1}, x_{2}, y\right) & =f_{\hat{X}_{1}}\left(x_{1}-\alpha_{P C} y\right) f_{\hat{X}_{2}}\left(x_{2}-\alpha_{M} y\right) f_{Y}(y) \\
& \times I\left\{0 \leq y \leq \min \left\{\frac{x_{1}}{\alpha_{P C}}, \frac{x_{2}}{\alpha_{M}}\right\}\right\}
\end{aligned}
$$

where $I\{S T\}=1$ if statement $\mathrm{ST}$ is true and $I\{S T\}=0$ otherwise.

In order to facilitate the double conversion of $\hat{w}_{V a r}(w, s)$ for numerical tractability, we assume that $\hat{X}_{1}(j)$ and $\hat{X}_{2}(j)$ are exponentially distributed with respective probability density functions given by

$$
f_{\hat{X}_{1}}\left(\hat{x}_{1}\right)=\lambda_{P C} e^{-\lambda_{P C} \hat{x}_{1}} \quad ; \quad f_{\hat{X}_{2}}\left(\hat{x}_{2}\right)=\lambda_{M} e^{-\lambda_{M} \hat{x}_{2}} .
$$

In addition, it is assumed that the available search time for each customer in each day is deterministic so that $Y_{j}=T$ for all $j$. This is equivalent to saying that the generalized probability density function of $Y$ is given by the delta function, that is $f_{Y}(y)=\delta(y-T)$, where the delta function is defined as the unit operator for convolution, that is, $u(x)=\int_{0}^{\infty} u(\tau) \delta(x-$ $\tau) d \tau$ for any integrable function $u(x)$. Since a day consists of 24 hours, which is deterministic, and since an ordinary consumer spends certain regular hours for sleeping and working every day, it is reasonable to assume that a fixed portion of a day can be devoted to information gathering through the 
Internet. This assumption facilitates the necessary inversion process. Under this assumption, Equation (38) can now be rewritten as

$$
\begin{aligned}
f_{\underline{X}, Y}\left(x_{1}, x_{2}, y\right) & =\lambda_{P C} e^{-\lambda_{P C}\left(x_{1}-\alpha_{P C} y\right)} I\left\{x_{1} \geq \alpha_{P C} y\right\} \\
& \times \lambda_{M} e^{-\lambda_{M}\left(x_{2}-\alpha_{M} y\right)} I\left\{x_{2} \geq \alpha_{M} y\right\} \delta(y-T) .
\end{aligned}
$$

Under these assumptions, we are now in a position to derive $\hat{\hat{w}}_{V a r}(w, s) / s$ for $\operatorname{Var} \in\{B, P C, M\}$. For notational convenience, the following functions are defined.

$$
\begin{gathered}
\zeta_{B}(w) \stackrel{\text { def }}{=} e^{-\alpha_{B} T w} \frac{\lambda_{P C}}{w+\lambda_{P C}} \cdot \frac{\lambda_{M}}{w+\lambda_{M}}, \text { where } \alpha_{B}=\alpha_{P C}+\alpha_{M} ; \\
\zeta_{P C}(w) \stackrel{\text { def }}{=} e^{-\alpha_{P C} T w} \frac{\lambda_{P C}}{w+\lambda_{P C}} \quad ; \quad \zeta_{M}(w) \stackrel{\text { def }}{=} e^{-\alpha_{M} T w} \frac{\lambda_{M}}{w+\lambda_{M}} .
\end{gathered}
$$

From Theorems 2 and 4 together with Equations (35) through (40), one has the following theorem. The proof demands careful implementation of integrals involving the delta function, but it is mechanical and so we decided to omit it here.

Theorem 8. (Series Representation of Double Laplace Transform of Search Completion Time)

For Var $\in\{B, P C, M\}$, let $\zeta_{V a r}(w)$ be as in Equation (41). Then $\hat{\hat{w}}_{\text {Var }}(w, s) / s$ is given by

$$
\frac{\hat{\hat{w}}_{V a r}(w, s)}{s}=\sum_{k=0}^{\infty}\left\{\frac{1}{s} \cdot e^{-(k+1) T s}\right\}\left\{\frac{1-\zeta_{V a r}(w)}{w} \zeta_{V a r}(w)^{k}\right\} .
$$

As discussed in Section 3, of central interest to this paper is the distribution function $W_{V a r}(z, t)$ of the search completion time. In order to evaluate $W_{V a r}(z, t)$ numerically, it is necessary to invert $\hat{\hat{w}}_{V a r}(w, s) / s$ with respect to both $w$ and $s$ based on Theorem 8. For doing so, we first obtain $b_{V a r}(z)$ by inverting $\zeta_{V a r}(w)$ in Equation (41). For $\operatorname{Var} \in\{B, P C, M\}$, let $h_{V a r}(z)$ be defined by

$$
h_{B}(z) \stackrel{\text { def }}{=} \int_{0}^{z} \lambda_{P C} e^{-\lambda_{P C}(z-x)} \lambda_{M} e^{-\lambda_{M} x} d x=\frac{\lambda_{P C} \lambda_{M}}{\lambda_{M}-\lambda_{P C}}\left(e^{-\lambda_{P C} z}-e^{-\lambda_{M} z}\right) ;
$$




$$
h_{P C}(z) \stackrel{\text { def }}{=} \lambda_{P C} e^{-\lambda_{P C} z} \quad ; \quad h_{M}(z) \stackrel{\text { def }}{=} \lambda_{M} e^{-\lambda_{M} z} .
$$

It should be noted that the Laplace transform of $h_{V a r}(z)$ corresponds to the portion of $\zeta_{V a r}(w)$ without the exponential function. Since the real domain form of the function $\exp \left\{-\alpha_{V a r} T w\right\}$ is given as $\delta\left(z-\alpha_{V a r} T\right)$, one can find $b_{V a r}(z)$ by convolving it with $h_{V a r}(z)$, yielding

$$
b_{V a r}(z)=h_{V a r}\left(z-\alpha_{V a r} T\right) I\left\{z \geq \alpha_{V a r} T\right\} .
$$

We also note that the Laplace transform $\left\{1-\zeta_{V a r}(w)\right\} / w$ corresponds to the survival function $\bar{B}_{V a r}(z)$ given by

$$
\bar{B}_{V a r}(z)=\int_{z}^{\infty} b_{V a r}(\tau) d \tau=\int_{\max \left\{z-\alpha_{V a r} T, 0\right\}}^{\infty} h_{V a r}\left(\tau-\alpha_{V a r} T\right) d \tau .
$$

We are now in a position to describe $W_{\text {Var }}(z, t)$ by inverting $\hat{\hat{w}}_{V a r}(w, s) / s$ based on Theorem 8 . It can be seen that $\hat{\hat{w}}_{\text {Var }}(w, s) / s$ is a separable function of $w$ and $s$. Since $\exp \{-(k+1) T s\} / s$ can be inverted as $U(t-(k+1) T)$ where $U(t)$ is the step function defined by $U(t)=I\{t \geq 0\}$, the summation for $\hat{\hat{w}}_{V a r}(w, s) / s$ based on Theorem 8 should be taken over $k$ in the range in which $t-(k+1) T \geq 0$.

For inverting the function of $w$ in $\hat{\hat{w}}_{V a r}(w, s) / s$, we denote the $k$-fold convolution of a function $r(z)$ with itself by $r^{(k)}(z)=\int_{0}^{z} r^{(k-1)}(z-\tau) r(\tau) d \tau$ for $k=1,2, \cdots$ with $r^{(0)}(z)=\delta(z)$. For the convolution of two different functions $r(z)$ and $q(z)$, we write $r * q(z)=\int_{0}^{z} r(z-\tau) q(\tau) d \tau$. With this notation, one finds from Equations (41) and (43) that

$$
b_{V a r}^{(k)}(z)=h_{V a r}^{(k)}\left(z-\alpha_{V a r} k T\right) I\left\{z \geq \alpha_{V a r} k T\right\}
$$

whose Laplace transform is given by $\zeta_{V a r}(w)^{k}$. The inversion of $\hat{\hat{w}}_{V a r}(w, s) / s$ with respect to $w$ can then be accomplished by convolving $b_{\text {Var }}^{(k)}(z)$ with $\bar{B}_{\text {Var }}(z)$ given in (44).

In summary, we have shown the following theorem. For notational convenience, we define

$$
k_{V a r}(z, T) \stackrel{\text { def }}{=}\left\lfloor\frac{z}{\alpha_{V a r} T}\right\rfloor \quad ; \quad k(t, T) \stackrel{\text { def }}{=}\left\lfloor\frac{t}{T}\right\rfloor,
$$

where $\lfloor a\rfloor$ is the largest integer which is less than or equal to $a$. 
Theorem 9. (Inversion of Double Laplace Transform of Search Completion Time into Real Domain)

For $\operatorname{Var} \in\{B, P C, M\}$, let $k_{V a r}(z, T)$ and $k(t, T)$ be as in (46). One then has

$$
W_{V a r}(z, t)=\sum_{k=0}^{\min \left\{k_{V a r}(z, T), k(t, T)\right\}} \bar{B}_{V a r} * b_{V a r}^{(k)}(z)
$$

In order to evaluate $W_{V a r}(z, t)$ in Theorem 9 , it is necessary to compute convolutions $b_{V a r}^{(k)}(z)$ and $\bar{B}_{V a r} * b_{V a r}^{(k)}(z)$ repeatedly. For this purpose, the Laguerre transform is employed. The Laguerre transform, originally developed by Keilson and Nunn (1979), Keilson et al. (1981), and further studied by Sumita (1981), maps a continuum function $f(x)$ into a sequence $\left(f_{n}^{\sharp}\right)$, thereby providing an algorithmic basis for computing a variety of continuum operations through lattice operations. For two such functions $f(x)$ and $g(x)$, for example, the convolution $f * g(x)$ can be mapped into the lattice convolution $(f * g)_{n}^{\sharp}=\sum_{k=0}^{n} f_{n-k}^{\sharp} g_{k}^{\sharp}$. The reader is referred to Sumita and Kijima $(1988,1991)$ for a succinct summary of theory and algorithms of the Laguerre transform.

\section{Numerical Examples}

We next illustrate how mobile access affects information search completion time and the conversion rate through numerical examples. The underlying parameter values for representing Type PC and M customers are shown in Table 2.The assumption in Equation (36) is satisfied.

Table 2: Basic Set of Parameter Values

\begin{tabular}{|c|c|c|c|c|c|c|c|c|c|}
\hline parameter & $\lambda_{P C}$ & $\lambda_{M}$ & $\alpha_{P C}$ & $\alpha_{M}$ & $z$ & $T$ & $N_{\text {Var }}$ & $K$ & $p$ \\
\hline value & 1.0 & 1.5 & 0.15 & 0.1 & 100 & 1.0 & 500 & 100 & 0.5 \\
\hline
\end{tabular}

For Type B customers, the use of a mobile device for information search through the Internet may replace the use of a PC. In order to reflect this point, we define the functional relationships among $\hat{\lambda}_{P C}, \hat{\lambda}_{M}, \hat{\alpha}_{P C}$ and $\hat{\alpha}_{M}$ for Type B customers as

$$
\hat{\lambda}_{P C}=\lambda_{P C}+\frac{1}{\hat{\lambda}_{M}} \quad ; \quad \hat{\alpha}_{P C}=\alpha_{P C} \cdot \frac{1}{1+\hat{\alpha}_{M}} .
$$




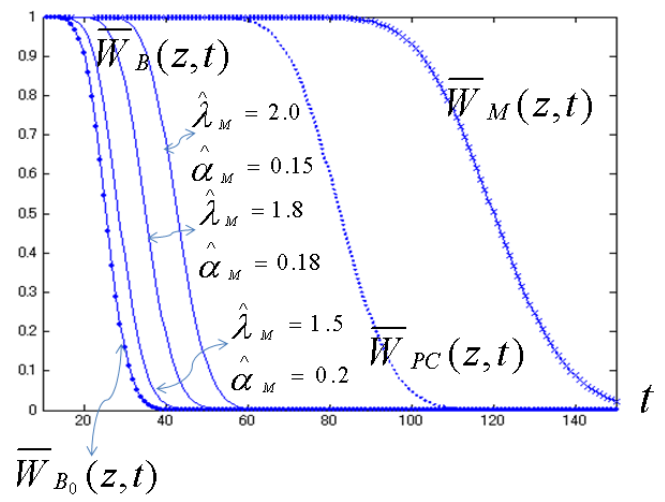

Figure 1: Survival Function of Information Search Completion Time

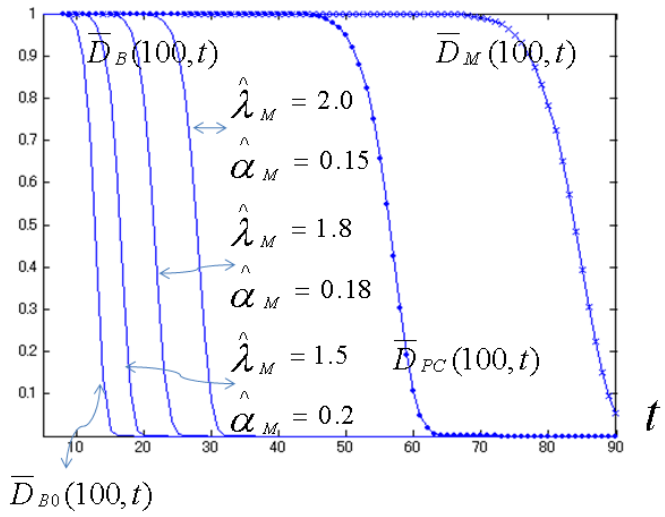

Figure 2: Survival Function of Time Required to Reach Conversion Rate of 1/15

Here, we consider a situation in which an original Type PC customer with $\lambda_{P C}$ and $\alpha_{P C}$ becomes a Type $\mathrm{B}$ customer by starting to use a mobile device. The degree of mobile device use is characterized by $\hat{\lambda}_{M}>0$ and $\hat{\alpha}_{M}>0$, which results in degrading the use of PC where $\hat{\lambda}_{P C}$ is increased from $\lambda_{P C}$ by adding $1 / \hat{\lambda}_{M}$, and $\hat{\alpha}_{P C}$ is decreased from $\alpha_{P C}$ by multiplying $1 /\left(1+\hat{\alpha}_{M}\right)$.

In Figure 1, the survival functions $\bar{W}_{V a r}(z, t)$ of the search completion time are plotted for $\operatorname{Var} \in\left\{B_{0}, B, P C, M\right\}$. Here, the right-most curve $\bar{W}_{M}(z, t)$ corresponds to Type $\mathrm{M}$ customers who employ mobile access exclusively. The next curve $\bar{W}_{P C}(z, t)$ describes the survival function for search 
completion time for Type PC customers who utilize the Internet only through the PC access. The left-most curve $\bar{W}_{B_{0}}(z, t)$ represents Type $B$ customers who use both the mobile access and the PC access without replacement effects at all between the two different accesses. The three curves $\bar{W}_{B}(z, t)$ in between correspond to Type B customers where mobile access replaces PC access to some extent based on Equation (47), and the effects of this replacement becomes weaker from the right to the left. The stochastic ordering among these search completion times is evident. For a user to complete information search with probability 0.7 or more, for example, 16 hours would be needed for a Type $B_{0}$ customer. This number increases from 16 to 18, 25, 34, 78 and 99 as the curve moves from the left to the right.

Figure 2 depicts similar curves for the time required to have 100 or more customers converted among a total population of 1,500. For achieving the conversion rate of $1 / 15$, with probability 0.7 or more, only 14 hours will be needed if all customers are of Type $B_{0}$. As before, this number increases from 14 to $16,21,27,55$ and 78 as the curve moves from the left to the right.

\section{Concluding Remarks}

As mobile devices become more sophisticated, it is important to find a way to assess the impact of mobile access to the Internet on e-commerce performance. We have studied the customer conversion rate in this research. We also developed a mathematical model for information search. Our approach enables assessment of the impact of mobile access on information search completion time and the customer conversion rate.

How to model information gathering from the Internet through both PC access and mobile access in a unified manner presented a new theoretical challenge. A typical user of the Internet would have duration of a day dedicated to information gathering. The information search completion time can then be defined as the time until the sum of the amounts of information gathered from the Internet through the two channels, possibly over multiple days, reaches a pre-specified threshold. Clearly, the duration of the Internet search in a day is limited in length, and therefore the amount of information gathered through PC access and that gathered through mobile access in a day would be not independent but correlated. Consequently, it became necessary to generalize the cumulative shock model associated with a renewal sequence proposed by Sumita and Shantikumar (1985) so that a sequence of shock vectors, rather than a sequence of shocks, could be incorporated. 
By overcoming this theoretical challenge, the model proposed in this paper provides a means to quantify the impact of mobile access to the Internet on the information search completion time and the customer conversion. For example, one may estimate how the customer conversion rate may be enhanced by improving the mobile access base so as to double the expected amount of information that could be gathered through each mobile access.

In order to apply the model proposed in this paper in a realistic environment, one has to define first the unit of information gathered from the Internet. Assuming that customers are interested in gathering information about a group of products, one way of defining the unit of information may be to introduce several categories of information about the product group, for example, prices, functions, designs, makers and brands of individual products in the group. For each category, the score of 1 through 10, possibly with appropriate weights, may be assigned, where the score of a category represents the information about the product group with respect to the category that can be absorbed in one visit to the website. Each website is represented by the sum of such scores across the categories, and the amount of information gathered through one Internet access can be defined as the sum of the scores of all the websites reached during the Internet access.

The set of websites containing information about at least one product in the product group may be decomposed into three classes: the set of websites $W E B_{P C}$ allowing $\mathrm{PC}$ access only, the set of websites $W E B_{M}$ accepting mobile access only, and the set of websites $W E B_{B}$ which can be reached through both $\mathrm{PC}$ access and mobile access. In our model, we recall that the amount of information gathered through PC access during the search period $Y_{j}$ on the $j$-th day is denoted by $X_{1}(j)$, while the counterpart for mobile access is defined as $X_{2}(j)$. It is assumed that both $X_{1}(j)$ and $X_{2}(j)$ consist of two parts: a part independent of $Y_{j}$ and another part proportional to $Y_{j}$. The former parts for $X_{1}(j)$ and $X_{2}(j)$ are denoted by $\hat{X}_{1}(j)$ and $\hat{X}_{2}(j)$ respectively. Within the context of the discussion above, $\hat{X}_{1}(j)$ and $\hat{X}_{2}(j)$ may represent the amount of information gathered from the landing website through PC access and that through mobile access respectively, while the second parts, which are assumed to be proportional to $Y_{j}$ with constants $\alpha_{P C}$ and $\alpha_{M}$, may describe the amount of information gathered from subsequent net surfing through $\mathrm{PC}$ access and mobile access respectively. Accepting the assumption that the $\hat{X}_{1}(j)$ and $\hat{X}_{2}(j)$ are exponentially distributed, the necessary parameter values $\lambda_{P C}, \lambda_{M}, \alpha_{P C}$ and $\alpha_{M}$ can be estimated by exam- 
ining the distributions of the scores of the websites in $W E B_{P C}, W E B_{M}$ and $W E B_{B}$ separately, along with the frequency distributions to those websites. A separate consumer survey would be needed so as to specify the threshold level $z$.

While the procedure for applying the model in a realistic environment can be logically described as above, the tasks involved in implementing the procedure could be laborious and cumbersome. Given a group of products, say digital cameras, it would not be easy to identify the three classes of websites $W E B_{P C}, W E B_{M}$ and $W E B_{B}$ with sufficient coverage to be meaningful for the study. Specifying information categories that would affect the purchasing decision of consumers significantly would also be nontrivial. Even more difficult is how to score individual websites with respect to such categories in a justifiable manner. The task of designing the consumer survey for estimating the threshold level $z$ and the information gathering time $Y$ per day would be also very tough.

This type of analytical research for understanding the impact of mobile access to the Internet is still in its infancy. Performance measures other than the conversion rate should be addressed. Furthermore, such performance analyses ought to be based on economic analysis. It also is necessary to establish a means to estimate the underlying parameters from real data, which would be quite challenging as discussed above. Despite such limitations concerning the realistic applicability of the mathematical model, it is definitely worth pursuing analytical research further through the management science approach because of the following three reasons.

1. Analytical models enable one to identify functional relationships among the key entities, which may not be observed easily through empirical studies alone.

2. Through extensive numerical experiments based on analytical models, one often can identify important qualitative properties that cannot be seen easily otherwise.

3. If analytical performance models are combined with economic analysis, certain strategic alternatives can be sorted in the order of their economic impact, even when the numbers derived from the analysis may not be necessarily reliable. 


\section{Acknowledgement}

The authors wish to express sincere gratitude to the Editor in Chief, Rob Kauffman, and the Guest Editor of the Special Issue, Kemal Altinkemer, who extended their exceptional generosity in time and energy so as to provide many helpful comments and suggestions that have contributed to improve the original version of this paper substantially. Without their help, the readability of this paper would have never reached the present level. Valuable comments by two anonymous referees are also greatly appreciated. In particular, some important changes are made based on their suggestions to incorporate the effect of information accumulation across different search periods and the possibility for mobile access to partially replace PC access.

\section{References}

[1] Barwise, P. TV, PC, or mobile? future media for consumer e-commerce. Business Strategy Review, 12, 1, 2001, 35-42.

[2] Chae, M., and Kim, J. What's so different about the mobile Internet. Communications of the ACM, 46, 12, 2003, 240-247.

[3] Hammond, K. B2C e-commerce 2000-2010: what experts predict. Business Strategy Review, 12, 1, 2001, 43-50.

[4] Dusan. B. Mobile web users to top 1.7 billion by 2013. Juniper Research, Basingstoke, Hampshire, 2008. Available at http://www.intomobile.com/2008/07/03/juniper-research-mobileweb-users-to-top.html. Last accessed on January 18, 2010.

[5] Dan, N. Mobile phone subscribers to top 4 billion by 2010. IDG News Service, Taipei, Taiwan, December 13, 2006. Available at http://www.computerworld.jp/news/mw/52310.html. Last accessed on Junuary 18, 2010.

[6] Khan, I., Weishaar, B., and Polinsky, L. State of the mobile Internet market. North America Equity Research, J.P.Morgan, New York, NY, 2008.

[7] Keilson, J., and Nunn, W.R. Laguerre transformation as a tool for the numerical solution of integral equations of convolution type. Applied Mathematics and Computation, 5, 4, 1979, 313-359. 
[8] Keilson, J., Nunn, W.R., and Sumita, U. The bilateral Laguerre transform. Applied Mathematics and Computation, 8, 2, 1981, 137-174.

[9] Kim, S.H. Impact of mobile-commerce: benefits, technological and strategic issues and implementation. Journal of Applied Sciences, 6, 12, 2006, 2523-2531.

[10] Neilsen Media Research. Critical Mass: the worldwide state of the mobile web, 2008. Available at http://www.nielsenmobile. com/documents/CriticalMass.pdf. Last accessed on Junuary 18, 2010.

[11] Okazaki, S., and Hirose, M. Effects of displacement-reinforcement between traditional media, PC Internet and mobile Internet: a quasiexperiment in Japan. International Journal of Advertising, 28, 1, 2009, 77-104.

[12] Osterman Research. The growing impact of mobile messaging. Black Diamond, Washington, 2007. Available at http://media. techtarget.com/searchMobileComputing/downloads/Mobile_ messaging impact.pdf. Last accessed on Junuary 18, 2010.

[13] Park, Y.H., and Fader, P.S. Modeling browsing behavior at multiple websites. Marketing Science, 23, 3, 2004, 280-303.

[14] Roto, V. Browsing on mobile phones. In Jana, R. and Wei, B. (eds.), Prodeedings of the MobEA III workshop on customer focused mobile services, May 2005, Chiba, Japan, Keio University, Chiba, Chiba, 2005.

[15] Roto, V. Search on mobile phones. Journal of the American Society for Information Science and Technology, 57, 6, 2006, 834-837.

[16] Siau, K., Sheng, H., and Nah, F,F. The value of mobile commerce to customers. In Zhang, P., Lazar, J., and McCoy, S. (eds.), Proceedings of the Third Annual Workshop on HCI Research in MIS, December 2004, Washington, DC, Association for Information Systems, Seattle, Washington, 2004.

[17] Sumita, U. Development of the Laguerre transform method for numerical exploration of applied probability models. Doctoral thesis, Graduate School of Management, University of Rochester, Rochester, NY, 1981. 
[18] Sumita, U., and Kijima, M. Theory and algorithms of the Laguerre transform, part I: Theory. Journal of the Operations Research Society of Japan, 4, 31, 1988, 467-494.

[19] Sumita, U., and Kijima, M. Theory and algorithms of the Laguerre transform, part II: Algorithm. Journal of the Operations Research Society of Japan, 4, 34, 1991, 467-494.

[20] Sumita, U., and Shanthikumar, J. G. A Class of Correlated Cumulative Shock Models. Advances in Applied Probability, 17, 2, 1985, 347-366 .

[21] Thompson, H. G., Jr, and Garbacz, C. Mobile, fixed line and Internet service effects on global productive efficiency. Information Economics and Policy, 19, 2, 2007, 189-214.

[22] Wu, J.H., and Hisa, T. L. Analysis of e-commerce Innovation and Impact: a hypercube model. Electronic Commerce Research and Applications, 3, 4, 2004, 389-404.

\section{Appendix}

\section{A. Analysis Approach: Overview and Explanation}

In order to model information gathering from the Internet through both PC access and mobile access in a unified manner, we first consider a customer who is to decide which product to buy or not to buy at all out of a product group, and begins to access the Internet for information search. The consumer can dedicate $Y_{j}$ time for the information search through both $\mathrm{PC}$ access and mobile access on the $j$-th day. The amount of information gathered through $\mathrm{PC}$ access during the search period $Y_{j}$ on the $j$-th day is denoted by $X_{1}(j)$, while the counterpart for mobile access is defined as $X_{2}(j)$, resulting in the total amount of information gathered during $Y_{j}$ being $Z_{B}(j)=X_{1}(j)+X_{2}(j)$. The customer continues this search until enough information is gathered to decide which product to buy or not to buy at all.

By surppressing the portion of the $j$-th day other than $Y_{j}$ for $j=1,2, \cdots$, we can define "time $t$ " to mean the cumulative amount of information search time given to the present. If we denote the number of days required to reach time $t$ by $N(t)$, the search completion time can be defined as the time until 
the sum, $S_{B}(t)=\sum_{j=1}^{N(t)} Z_{B}(j)$, first exceeds a pre-specified threshold $z$. This information search procedure is depicted in Figure 3.

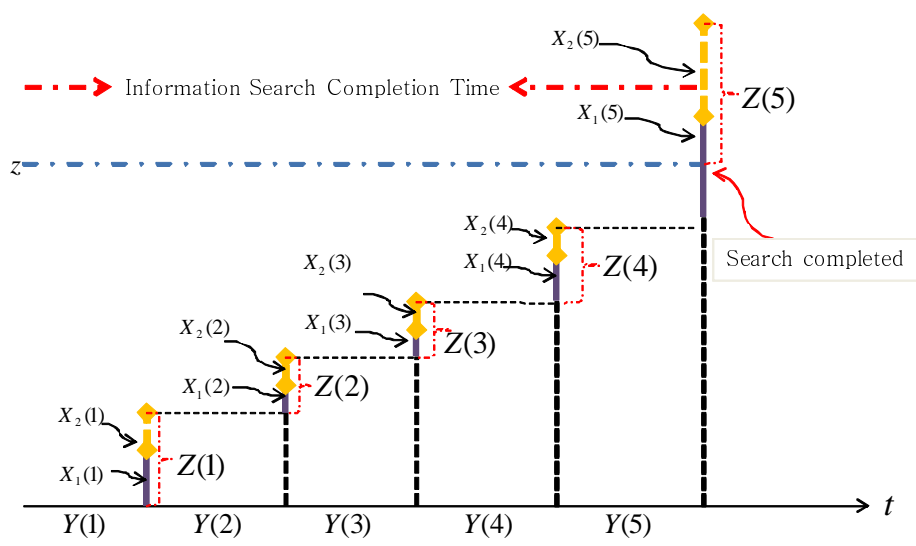

Figure 3: Information Search via PC Access and Mobile Access to the Internet

A mathematical difficulty arises from the reality that $X_{1}(j), X_{2}(j)$ and $Y_{j}$ should be correlated. This means that the more one collects information from the Internet via $\mathrm{PC}$ access, the less one does so via mobile access, and vice versa, since the length of $Y_{j}$ is limited. However, it may be reasonable to assume that $\left[X_{1}(j), X_{2}(j), Y_{j}\right]$ can be treated as a sequence of independent random vectors, since information search today may not be affected so much by information search yesterday. By taking advantage of this, the mathematical model enjoys the regenerative structure in that, if $S_{B}(t)$ is less than $z$ and therefore the information search is not yet completed at time $t$, then $S_{B}(\tau)$ is less than $z$ and so is $S_{B}(\tau)+S_{B}(t-\tau)$ for any $\tau, 0 \leq \tau \leq t$. This recursive structure enables one to solve a functional equation for finding the distribution of $S_{B}(t)$ based on the Laplace transform analysis. This distribution, in turn, enables one to derive the distribution of the search completion time, since $S_{B}(t)$ is less than $z$ if and only if the search completion time is greater than $t$.

\section{B. Notation}


Table 3. Notational Summary

\begin{tabular}{|c|c|c|}
\hline Notation & Definition & Comments \\
\hline$\underline{X}$ & $\underline{\underline{X}} \underline{\underline{X}}=\left[X_{1}, \cdots, X_{K}\right]$ & $\begin{array}{l}\text { a random vector with the } k \text {-th } \\
\text { component } X_{k}, k=1, \cdots, K\end{array}$ \\
\hline$A \stackrel{\text { def }}{=} B$ & A is defined by $B$ & \\
\hline $\inf \{\cdot\}$ & the infimum of $\{\cdot\}$ & for example: $\inf \{t: 1<t\}=1$ \\
\hline $\min \{\cdot\}$ & the minimum of $\{\cdot\}$ & for example: $\min \{1,2\}=1$ \\
\hline $\max \{\cdot\}$ & the maximum of $\{\cdot\}$ & for example: $\max \{1,2\}=2$ \\
\hline $\operatorname{Re}(\mathrm{s})$ & real part of a complex number $\mathrm{s}$ & for example: $\operatorname{Re}(x+i y)=x$ \\
\hline$\delta(x)$ & $f(x)=\int_{0}^{\infty} \delta(x-y) f(y) d y$ & known as the delta function \\
\hline$I(S T)$ & $\begin{array}{l}I(S T)=1 \text { if } \mathrm{ST} \text { is true, } \\
\text { and } I(S T)=0 \text { otherwise }\end{array}$ & known as the indicator function \\
\hline$U(x)$ & $\begin{array}{l}U(t)=I\{t \geq 0\} \\
\text { where } I \text { is defined above }\end{array}$ & known as the unit step function \\
\hline$X \succ_{s t .} Y$ & $P[X>x] \geq P[Y>x]$ & $\begin{array}{l}\text { the stochastic ordering } \\
\text { between } X \text { and } Y\end{array}$ \\
\hline$r * q(z)$ & $r * q(z)=\int_{0}^{z} r(z-\tau) q(\tau) d \tau$ & $\begin{array}{l}\text { the convolution of two } \\
\text { functions } r(z) \text { and } q(z)\end{array}$ \\
\hline$\lfloor x\rfloor$ & $\begin{array}{l}\text { the largest integer smaller } \\
\text { than or equal to } x\end{array}$ & $\begin{array}{l}\text { known as the floor function, } \\
\text { for example: }\lfloor\pi\rfloor=3\end{array}$ \\
\hline
\end{tabular}

